\title{
Role of the Programmed Death-1 (PD-1) pathway in regulation of Theiler's murine encephalomyelitis virus-induced demyelinating disease
}

\author{
Sho Takizawa a ${ }^{a}$, Tomoki Kaneyama a , Sayaka Tsugane a , Naoya Takeichi ${ }^{\text {a }}$, Satoshi Yanagisawa a , \\ Motoki Ichikawa ${ }^{a}$, Hideo Yagita ${ }^{\mathrm{b}}$, Byung S. Kim ${ }^{\mathrm{c}}$, Chang-Sung Koh ${ }^{\mathrm{a}, *}$
}

a Department of Biomedical Laboratory Sciences, Graduate School of Medicine, Shinshu University, Matsumoto, Nagano 390-8621, Japan

b Department of Immunology, Juntendo University School of Medicine, Bunkyo-ku, Tokyo 113-8421, Japan

c Department of Microbiology-Immunology, Northwestern University Finberg Medical School, 303 East Chicago Avenue, Chicago, IL 60611, USA

\section{A R T I C L E I N F O}

\section{Article history:}

Received 16 April 2014

Received in revised form 10 June 2014

Accepted 19 June 2014

\section{Keywords:}

Programmed Death-1 (PD-1)

Theiler's murine encephalomyelitis virus-

induced demyelinating disease (TMEV-IDD)

Multiple sclerosis (MS)

Demyelination

PD-L1

\begin{abstract}
A B S T R A C T
Programmed death-1 (PD-1) belongs to the CD28 family of co-stimulatory and co-inhibitory molecules and regulates adaptive immunity. This molecule induces the development of regulatory $\mathrm{T}$ cells, $\mathrm{T}$ cell tolerance, or apoptosis. We examined the role of PD-1 pathway in Theiler's murine encephalomyelitis virus (TMEV)-induced demyelinating disease (TMEV-IDD) mice. Up-regulation of PD-1 and PD-1 ligand-1 (PD-L1) mRNA expression in bone marrow-derived dendritic cells were induced by TMEV infection in vitro. Furthermore, PD-1 and PDL1 mRNA expression was increased in the spinal cords of the TMEV-infected mice in vivo. Treatment with a blocking monoclonal antibody $(\mathrm{mAb})$ against $\mathrm{PD}-1$, especially during the effector phase, resulted in significant deterioration of the TMEV-IDD both clinically and histologically. Flow cytometric analysis revealed a dramatically increase of $\mathrm{CD}^{+}{ }^{+} \mathrm{T}$ cells producing Th1 cytokines such as IFN- $\gamma$ and TNF- $\alpha$ in the spinal cord of anti-PD- $1 \mathrm{mAb}-$ treated mice.

These results indicate that the PD-1 pathway plays a pivotal regulatory role in the development of TMEV-IDD.
\end{abstract} (c) 2014 Elsevier B.V. All rights reserved.

\section{Introduction}

Multiple sclerosis (MS) is an immune-mediated chronic inflammatory demyelinating disease of the central nervous system (CNS) in humans (Wekerle, 1991). Although the etiology of MS is not clarified yet, epidemiological studies and investigations with experimental animal models have supported a potential role for viruses as the environmental trigger in disease induction (Allen and Brankin, 1993). Thus, a similar demyelinating disease induced by a virus could be one of the most attractive animal models in the study of the pathogenesis of MS. Theiler's murine encephalomyelitis viruses (TMEV), members of the genus Cardiovirus in the family of Picornaviridae, are natural enteric pathogens that cause CNS disease in mice (Pevear et al., 1987). Intracerebral (i.c.) injection of TMEV into susceptible strains of mice results in a chronic, progressive demyelinating disease characterized histologically by perivascular inflammatory cell infiltrates and primary demyelination of the CNS (Lipton and Dal Canto, 1976). The clinical signs of TMEVinduced demyelinating disease (TMEV-IDD) include a spastic waddling gait, extensor spasm, and incontinence (Lehrich et al., 1976). TMEV-IDD is considered an infectious mouse model for MS because the disease displays histopathologic, genetic, and clinical similarities to human MS (Dal Canto and Lipton, 1975; Nathanson and Miller, 1978; Kurtzke and

\footnotetext{
* Corresponding author. Tel.: +81263 37 3431; fax: +81263 373430 .

E-mail address: kshosei@shinshu-u.ac.jp (C.-S. Koh).
}

Hyllested, 1986; Kappel et al., 1991). The demyelination is linked to persistent TMEV infection in the CNS (Lipton et al., 1991), and histological findings are characterized by perivascular inflammatory cell infiltrates and primary demyelination in CNS (Lehrich, Arnason, 1976; Lipton et al., 1984). TMEV persists in the white matter of the spinal cord, mainly in microglia/macrophages, but also in astrocytes and oligodendrocytes throughout the life of a mouse (Lipton et al., 1984; Lipton et al., 1995). Persistent CNS virus infection in susceptible mouse strains triggers clonal expansion and differentiation of TMEV-specific T cells that are poorly controlled by normal immunoregulatory mechanism. Activated Th1 cells release proinflammatory cytokines in the CNS, resulting in the subsequent recruitment and activation of mononuclear phagocytes that initiate myelin destruction via both direct and bystander mechanisms (Miller et al., 1997a; Kim et al., 1998). Furthermore, approximately 4 weeks after onset of clinical disease, i.e., 8 weeks post infection, T-cell responses to myelin epitopes arise in an ordered temporal progression (Miller et al., 1997b) consistent with a role for both virusand myelin-specific responses in the chronic phase of the disease.

The immune system has the difficult challenge of discerning and defending against a diversity of microbial pathogens, while simultaneously avoiding self-reactivity. While central tolerance mechanisms result in deletion of the majority of self-reactive T lymphocytes, some $\mathrm{T}$ cells specific for self-antigens escape into the periphery (van Noort et al., 1993; Lohmann et al., 1996). To further control the development of autoimmunity, multiple mechanisms of peripheral tolerance have 
evolved, including T-cell anergy, deletion, and suppression by regulatory $T$ cells (Tregs). Failure of any of these tolerance mechanisms can result in autoimmune disease. T-cell co-stimulatory pathways play critical roles in regulating the delicate balance between protective immunity and tolerance. The critical immunoregulatory function of T-cell co-stimulation has led to significant advances in the identification and characterization of T-cell co-stimulatory pathways (Sharpe, 2009). Now, it is well known that co-stimulatory pathways can provide positive second signals that promote T-cell activation as well as negative co-inhibitory signals that suppress T-cell responses, mediate T-cell tolerance, and prevent autoimmunity. In addition, co-stimulatory and co-inhibitory pathways not only regulate responses of naive T cells but can also control effector, memory, and Tregs. Thus, the functions of these pathways in regulating T-cell activation and tolerance have expanded. Co-inhibitory pathways can control effector T-cell responses and the development and function of Treg cells as well as the fate of naive $T$ cells upon antigen encounter. The pathway consisting of programmed death-1 (PD-1) receptor (CD279) and its ligands, PD-L1 (B7-H1; CD274) and PD-L2 (B7-DC; CD273), delivers inhibitory signals that regulate the balance among $\mathrm{T}$-cell activation, tolerance, and immune-mediated tissue damage (Okazaki and Honjo, 2007; Sharpe et al., 2007; Nurieva et al., 2009). This pathway exerts critical inhibitory functions in the setting of persistent antigenic stimulation such as during encounter of self-antigens, chronic viral infections, and tumors (Sharpe, Wherry, 2007; Keir et al., 2008).

Because the PD-1 pathway plays a crucial role in regulating autoimmunity by negatively controlling $\mathrm{T}$ cell activation as well as cytolytic $\mathrm{CD}^{+} \mathrm{T}$ cell function, we have investigated the role of the PD- 1 pathway in TMEV-IDD. Here, we describe for the first time that blockade of PD-1 pathway by a blocking monoclonal antibody ( $\mathrm{mAb}$ ) against PD- 1 in the effector phase markedly exacerbated the disease progression of TMEVIDD.

\section{Materials and Methods}

\subsection{Mice}

Female SJL/J mice, 6 weeks old, were purchased from the Charles River Laboratories Japan, Inc. (Ibaraki, Japan), housed and cared for in an approved facility, in accordance with the Shinshu University Guide for Laboratory. The animals were kept in aluminum cages containing pine chips, and given food and water ad libitum.

\subsection{Virus}

The BeAn strain of TMEV was expanded in Baby Hamster Kidney (BHK) cell monolayers in DMEM supplemented with 7\% donor calf serum. Cell lysates with known Plaque Forming Unit (PFU) were used as viral stock for animal experiments. Partially purified virus was prepared following centrifugation through $30 \%$ sucrose as previously described and used for in vitro assays (Yauch et al., 1998). Viral titer was determined by the standard plaque assay on BHK cells.

\subsection{Infection of mice with virus}

Demyelinating disease was induced by injection of $1 \times 10^{6} \mathrm{PFU}$ TMEV in $30 \mu \mathrm{l}$ of PBS into the left cerebral hemisphere of female SJL/J mice after anesthetization. Intracerebral (i.c.) injection with $1 \times 10^{6}$ PFU consistently induces chronic gait abnormalities and neurologic signs in greater than $90 \%$ of $\mathrm{SJL} / \mathrm{J}$ mice. Clinical symptoms of the disease were assessed alternate-day on the following grading scale: grade $0=$ no clinical signs; grade $1=$ mild waddling gait; grade $2=$ moderate waddling gait and hindlimb paresis; grade $3=$ severe hindlimb paralysis; grade $4=$ severe hindlimb paralysis and loss of righting reflex; grade $5=$ moribund.

\subsection{Generation of bone marrow-derived dendritic cells (BMDCs)}

Bone marrow (BM) cells were harvested from uninfected SJL/J mice, passed through nylon mesh and depleted of erythrocytes with RBC lysis buffer. $1 \times 10^{7}$ BM cells were seeded in 10-cm dish in $10 \mathrm{ml}$ of RPMI 1640-based complete medium supplemented with $20 \mathrm{ng} / \mathrm{ml} \mathrm{GM-CSF}$ (Wako, Oska, Japan). The cultures were usually fed every 2 days, aspirating $75 \%$ of the medium, and adding back fresh medium with GMCSF. After 8 days, loosely adherent cells were collected and incubated with TMEV (MOI: multiplicity of infection $=10$ ) for $24 \mathrm{~h}$.

\subsection{Treatment with anti-PD-1 $m A b$}

Six weeks old female SJL/J mice were separated into groups (A-C; Table 1). TMEV was injected into SJL/J mice i.c. at day 0. Control rat IgG (Sigma Aldrich) anti-PD-1 mAb (RMP1-14) (Yamazaki et al., 2005) was injected intraperitoneally (i.p.) into mice on $-3,0,3,6,9$, 12 days after i.c. infection with TMEV at the induction phase or 17, 20, $23,26,29,32$ days after i.c. infection with TMEV at the effector phase with a dose of $250 \mu \mathrm{g}$ in a volume of $100 \mu \mathrm{l} /$ mouse per injection. Details of the experimental design are given in Table 1.

\subsection{Isolation of infiltrating cells from spinal cord}

Single-cell suspensions were prepared according to standard methods. Briefly, Percoll-NaCl solution was prepared by addition of 9 part (v/v) of Percoll (GE Healthcare) to 1 part ( $/ / \mathrm{v}$ ) of $1.5 \mathrm{M} \mathrm{NaCl}$. The spinal cord cell suspensions were centrifuged at $3000 \mathrm{~g}$ for $5 \mathrm{~min}$ and resuspended in $5 \mathrm{ml}$ of $37 \%$ Percoll- $\mathrm{NaCl}$ solution diluted with culture medium and overlaid by $2.5 \mathrm{ml}$ of $70 \%$ Percoll- $\mathrm{NaCl}$ solution diluted with culture medium. The gradient was centrifuged at $2000 \mathrm{~g}$ for $20 \mathrm{~min}$ and the mononuclear cells were harvested from the $37 \%-70 \%$ interface, washed, and counted.

\subsection{Flow cytometry}

For intracellular staining, cells were isolated from spleen and spinal cord. Cells were stimulated in culture medium with PMA (50 ng/ml; Wako pure chemical), Calcinomycin A23187 ( $1 \mu \mathrm{g} / \mathrm{ml}$ : Sigma), and Brefeldin ( $10 \mu \mathrm{g} / \mathrm{ml}$; Sigma) for $6 \mathrm{~h}$ at $37{ }^{\circ} \mathrm{C}$, in a humidified $5 \% \mathrm{CO}_{2}$ atmosphere. Cells were washed and triple-labeled with PerCP-conjugated anti-mouse CD4 mAb, PE-conjugated anti-mouse IL-4, IL-10 or IL-17 $\mathrm{mAb}$, and FITC-conjugated anti-mouse IFN- $\gamma$ or TNF- $\alpha \mathrm{mAb}$ for $30 \mathrm{~min}$ at room temperature in dark (all antibodies were obtained from BD Biosciences, Japan). After two steps of wash, cells were analyzed on FACSCalibur and FACSCan flow cytometers (BD Biosciences).

\subsection{Histology}

In each experiment, mice were blindly selected from each group beforehand for histological examination and sacrificed on day 34 post i.c. infection. Spinal cords were removed and fixed in $4 \%$ paraformaldehyde in PBS, pH 7.4. Spinal cord sections from paraffin-embedded tissues were prepared at 3-um thickness. Sections were stained with hematoxylineosin or Kluver-Barrera's staining.

\subsection{Gene expression measurements}

Total RNA was extracted from spleen and spinal cord using TRIzol (Invitrogen) following the manufacturer's instructions. Prior to the reverse transcription, potentially contaminating residual genomic DNA was eliminated with DNase I (Roche). RNA was reverse transcribed using SuperScript ${ }^{\mathrm{TM}}$ III. Messenger RNA (mRNA) was quantified by real-time RT-PCR using a TaqMan Fast Universal Master Mix (Applied Biosystems) in an Applied Biosystems StepOnePlus ${ }^{\mathrm{TM}}$ real-time PCR System (Applied Biosystems) with the following probe, sense primer, 
Table 1

Summary of effects of anti-PD-1 mAb administration on TMEV-IDD.

\begin{tabular}{|c|c|c|c|c|c|c|}
\hline Group & Treatment & Number of mice & Date of Treatment & $\mathrm{MDO}^{\mathrm{a}}+\mathrm{SD}$ (day) & $\mathrm{MMS}^{\mathrm{b}} \pm \mathrm{SD}$ & $\mathrm{MCS}^{\mathrm{c}} \pm \mathrm{SD}$ \\
\hline A & Control rat IgG & 15 & Day $17,20,23,26,29,32$ (effector phase) & $23.7 \pm 5.0$ & $2.4 \pm 1.4$ & $10.8 \pm 8.4$ \\
\hline B & anti-PD-1 mAb & 15 & Day $-3,0,3,6,9,12$ (induction phase) & $22.8 \pm 2.2$ & $2.2 \pm 1.2$ & $10.1 \pm 6.6$ \\
\hline C & anti-PD-1 mAb & 18 & Day $17,20,23,26,29,32$ (effector phase) & $20.9 \pm 3.1^{*}$ & $3.3 \pm 1.4^{*}$ & $19.2 \pm 9.2^{*}$ \\
\hline
\end{tabular}

'Significant difference between Group A and B ( $p<0.05)$ MDO: Mean Day of Onset.

MMS: Mean maximum clinical score.

MCS: Mean cumulative clinical score.

and anti-sense primer: TMEV (5'-CGCGCGCCCAAAAGCAAGC-TAMRA$3^{\prime}$, and 5'-TTGAGCTCTCTGAGGGTGAACA-3' and 5'-CTAGAACCTTCCCG CCTCCTT-3'). The primers for PD-1 (Mm00435532_m1), PD-L1 (Mm00452054_m1) and $\beta$-actin (Mm01205647_g1) were obtained from Applied Biosystems. Amplification was conducted in a total volume of $10 \mu \mathrm{l}$ for 50 cycles of $1 \mathrm{sec}$ at $95{ }^{\circ} \mathrm{C}$ and $20 \mathrm{sec}$ at $60{ }^{\circ} \mathrm{C}$. mRNA levels were determined after normalization of mRNA concentration against $\beta$-actin.

\subsection{Statistical analysis}

Statistical comparison among groups was performed with MannWhitney U-test using JMP software (SAS institute Inc, Tokyo, Japan). Data was considered statistically significant when the p-value was $<0.05$.

\section{Results}

\subsection{TMEV induces PD-1 and PD-L1 in BMDCs}

The identification of altered expression of PD-1 and PD-L1 during TMEV infection provides initial data for justifying their role during disease. To determine the levels of PD-1 and PD-L1 expression upon TMEV infection, freshly grown and isolated BMDCs were infected with TMEV (MOI = 10) and assessed for PD-1 and PD-L1 mRNA expression by real-time RT-PCR analyses (Fig. 1). The data indicated that, compared with uninfected DCs, TMEV infection significantly induced the upregulation of both PD-1 and PD-L1 ( $\left.{ }^{*} \mathrm{p}<0.05\right)$.

\subsection{Expression of PD-1 and PD-L1 in spinal cord}

We further analyzed the expression of PD-1 and PD-L1 in the spinal cord at different time points after infection with TMEV by real-time RTPCR. The expression of PD-1 and PD-L1 significantly increased at day 10 , 20,30 , and 40 compared to day 0 post infection (Fig. 2). This suggested that intracerebral infection with TMEV induced persistent up-regulation of the PD-1 pathway signaling.

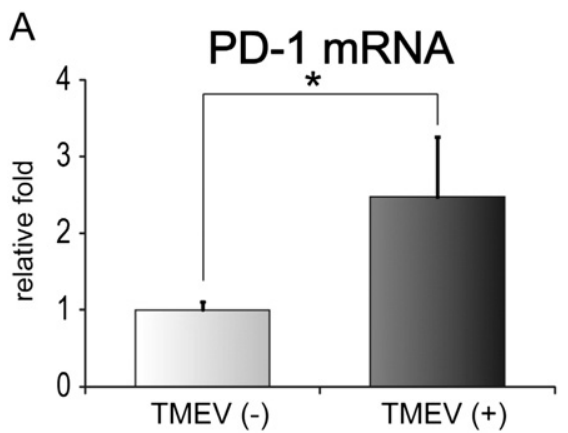

3.3. Treatment with anti-PD-1 mAb exacerbates the development of TMEV-IDD

The experimental results of anti-PD-1 mAb treatment are summarized in Table 1 and Fig. 3. Control IgG-treated animals showed the typical disease course of TMEV-IDD. Mean maximum clinical score (MMS) of the control group A was 2.4. MMS of the mice (group B) treated with anti-PD-1-mAb at the induction phase was 2.2. In contrast, MMS of the mice (group C) treated with anti-PD-1 mAb at the effector phase was significantly increased to $3.3\left({ }^{*} p<0.05\right)$. Similarly, mean cumulative clinical score (MCS) of the control group was 10.8. MCS of the mice in group B was 10.1. In contrast, clinical symptoms were markedly elevated in animals treated with anti-PD-1 $\mathrm{mAb}$ at the effector phase (group C) $\left({ }^{*} p<0.05\right)$ of which MCS was 19.2. Therefore, the PD-1-mediated signals appear to play an important role in preventing the development of TMEV-IDD particularly during the effector phase. Hereafter, we performed comparative study between the mice treated with anti-PD$1-\mathrm{mAb}$ and control IgG.

\subsection{Histologic findings}

At day 34, the disease severity reached nearly maximum (grade 4 or 5 ) in some mice. Therefore, representative mice were blindly selected from each group at day 34 for histological examination. Mild perivascular mononuclear cell (MNC) infiltration and demyelination were observed in the white matter of spinal cord from the control IgG-treated mice and the mice treated with anti-PD-1 mAb at induction phase (Fig. 4A, $\mathrm{B}, \mathrm{D}, \mathrm{E})$ which are the characteristics of TMEV-IDD. In contrast, severe inflammatory MNCs and demyelination were observed in the white matter of spinal cord from the mice treated with anti-PD-1 mAb at effector phase (Fig. 4C, F).

3.5. Treatment with anti-PD-1 $m A b$ has no significant effect on viral persistence

To determine the effect of anti-PD- $1 \mathrm{mAb}$ on TMEV replication in spinal cords, viral RNA levels in spinal cords of mice at 34 days post-

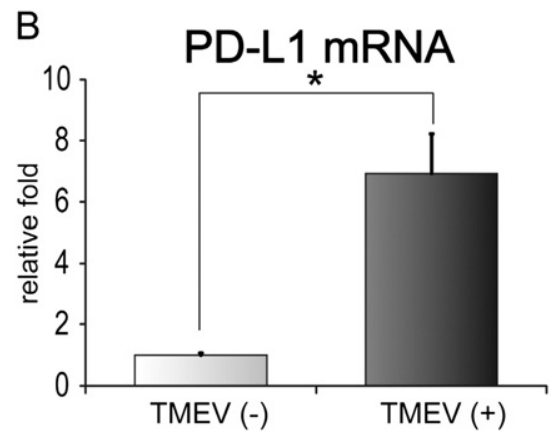

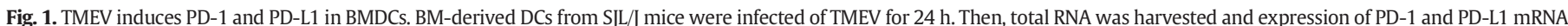

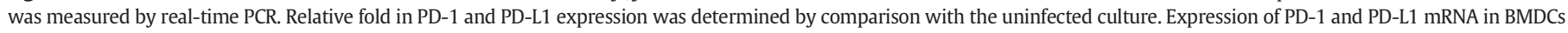
infected with TMEV was significantly increased compared with the uninfected culture $\left({ }^{*} \mathrm{p}<0.05\right)$. 
A

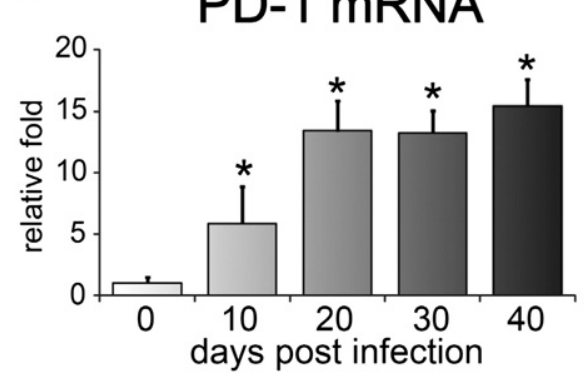

B

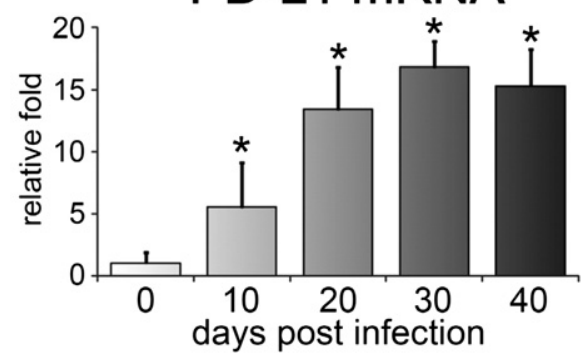

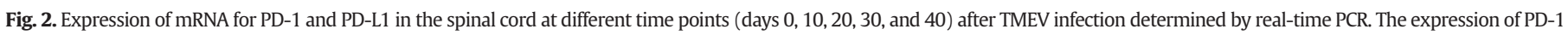


$\left({ }^{*} \mathrm{p}<0.05\right)$

infection were assessed using real-time RT-PCR. TMEV RNA levels were not significantly different between anti-PD-1 mAb-treated mice and control IgG-treated mice (Fig. 5). These results suggest that the blockade of PD-1-mediated signals inhibited the development of TMEV-IDD without significantly hindering TMEV persistence.

\subsection{Effect of anti-PD-1 $\mathrm{mAb}$ on the production of cytokines}

To examine the effect of anti-PD-1 $\mathrm{mAb}$ on the production of immune cytokines, we assessed the production of IFN- $\gamma$, TNF- $\alpha$, IL-4, IL10 , IL-17 by spleen cells and cells from spinal cords at day 34 postinfection. Numbers of cytokine-producing CD4 positive cells were analyzed by flow cytometry. The proportions of IFN- $\gamma$ or TNF- $\alpha$ producing cells were significantly increased in spleen and spinal cord from the mice treated with anti-PD-1 mAb at effector phase (Figs. 6 and 7). The percentage of IFN- $\gamma$ producing cells and IL-17 producing cells are very small around $1 \%$ in the spleen of mice with TMEV-IDD as published before (Takeichi et al., 2010; Yanagisawa et al., 2010; Tsugane et al., 2012). The percentage of IFN- $\gamma$ producing cells and IL-17 producing cells were significantly increased in the spleen of TMEV-infected and anti-PD-1 $\mathrm{mAb}$ treated mice from $0.51 \%$ to $1.77 \%$ ( $\mathrm{p}<0.05$ ) and from $1.18 \%$ to $1.54 \%(\mathrm{p}<0.05)$ respectively. These results suggest that the cells

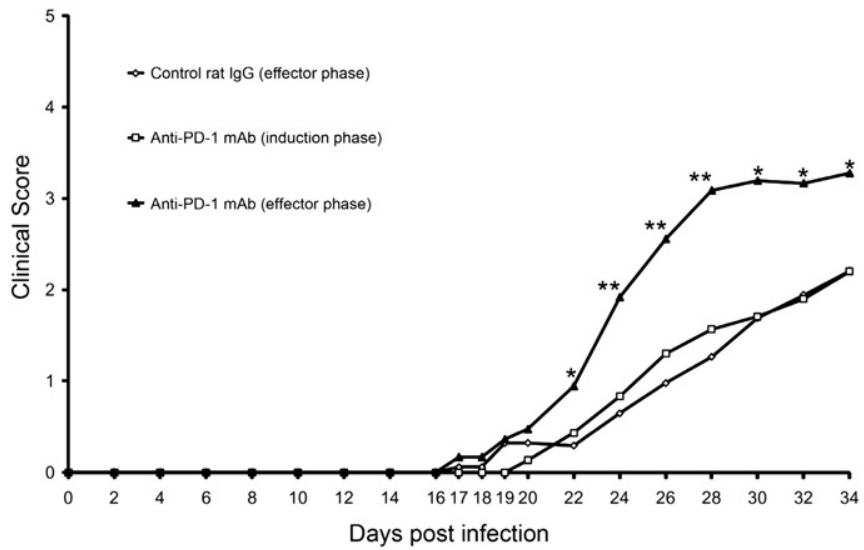

Fig. 3. Effect of anti-PD-1 mAb treatment on the clinical course of TMEV-IDD. This figure shows the summary of the clinical course of anti-PD- $1 \mathrm{mAb}$ treatment experiments. All mice were clinically observed from day 0 to day 34 post infection. Control rat IgG or anti-PD-1 mAb were injected i.p. on days $-3,0,3,6,9$, and 12 in the induction phase or days $17,20,23,26,29$, and 32 in the effector phase after i.c. infection of TMEV. Treatment design is given in Table 1. Clinical signs of demyelinating disease were significantly exacerbated $\left({ }^{*} \mathrm{p}<0.05 ;{ }^{* *} \mathrm{p}<0.01\right)$ in mice treated with anti-PD-1 mAb in the effector phase from day 22 to day 34 post infection, as compared with control mice. producing inflammatory cytokines were increased in the mice treated with anti-PD-1 mAb at effector phase, which might be responsible for the exacerbated TMEV-IDD.

\section{Discussion}

In this study, we examined the role of PD-1 pathway in the development of TMEV-IDD. We showed that administration of blocking $\mathrm{mAb}$ to PD-1 exacerbated the clinical signs of TMEV-IDD. After i.c. inoculation with TMEV, an initial viremia is followed by a persistent low level of CNS infection (Lipton et al., 1984). TMEV is known to infect neurons, glial cells, and macrophages in the spinal cord (Brahic et al., 1981; Clatch et al., 1990). Antigen presentation is mediated by either macrophages or glial cells, which contain TMEV antigens. As a consequence of the immunological process, major histocompatibility complex (MHC) class II restricted TMEV-specific delayed-type hypersensitivity (DTH)-mediating $\mathrm{CD} 4^{+}$T cells are activated, which in turn participate in immune-mediated inflammatory responses. It has been proposed that virus-specific DTH results in the recruitment and accumulation of macrophages in the CNS, producing demyelination via a nonspecific "bystander" mechanism (Clatch et al., 1986). Myelin damage induced following TMEV infection is an immune-mediated process. In susceptible mice, the persistence of viral antigens within the CNS may result in continuous activation of $T$ cells that can mediate pathogenesis through locally produced cytokines or direct cytolytic mechanisms. The role of $\mathrm{T}$ lymphocytes in immune-mediated demyelination has been confirmed by the fact that thymectomized, irradiated, and bone marrow-restored SJL/J mice fail to develop any clinical signs of TMEVIDD (Gerety et al., 1994). It has been shown that treatment of susceptible mice with blocking Abs against CD4 or MHC class II molecules can significantly suppress the development of inflammation and demyelination after infection with TMEV (Rodriguez et al., 1986; Friedmann et al., 1987; Welsh et al., 1987; Gerety, Rundell, 1994). Demyelination correlates well with the development of a chronic, virus-specific DTH response (Clatch, Lipton, 1986). In addition, TMEV-IDD is inhibited after induction of virus-specific tolerance, which downregulates Th1-type responses (Peterson et al., 1993; Karpus et al., 1995), and Th1-type cells specific for VP1 $233-250$ and VP2 $2_{74-86}$ were further shown to be involved in the pathogenesis of viral demyelination induced by TMEV (Yauch, Palma, 1998). These studies strongly suggest that virus-specific Th1 cells are the major component in myelin destruction by TMEV infection. The activation and differentiation of $\mathrm{T}$ cells require both antigen/MHC recognition and costimulatory signals. The costimulatory signals are provided by binding of specific receptors on $\mathrm{T}$ cells to their ligands on antigen-presenting cells (APCs) and are necessary for functional T cell activation (Williams and Unanue, 1990).

PD-1 has been described as a negative regulator of immunity that limits T- and B-cell function. PD-1 is a cell surface molecule expressed 

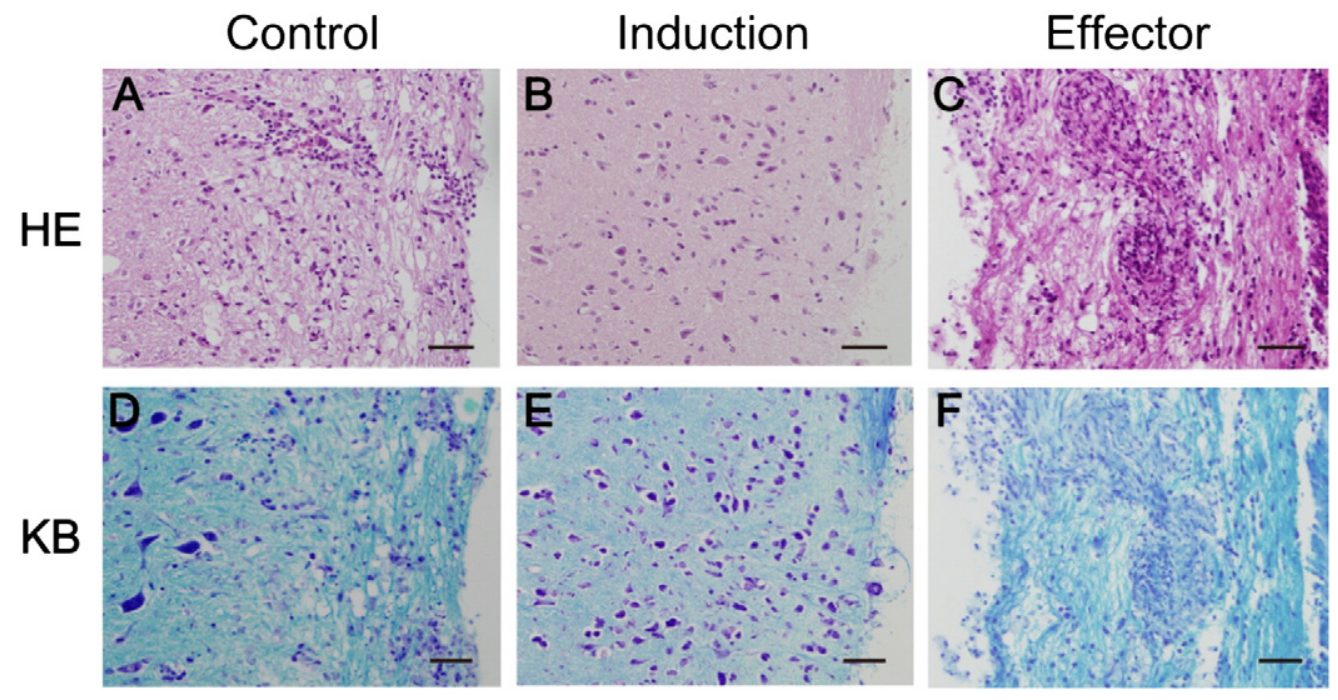

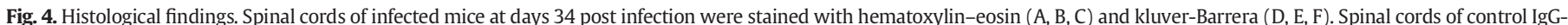

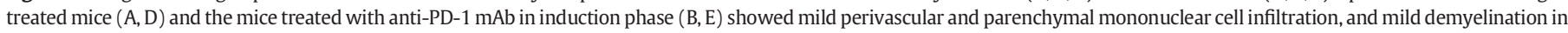

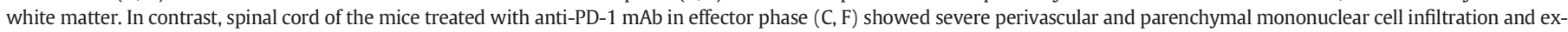
tensive demyelinative lesion.

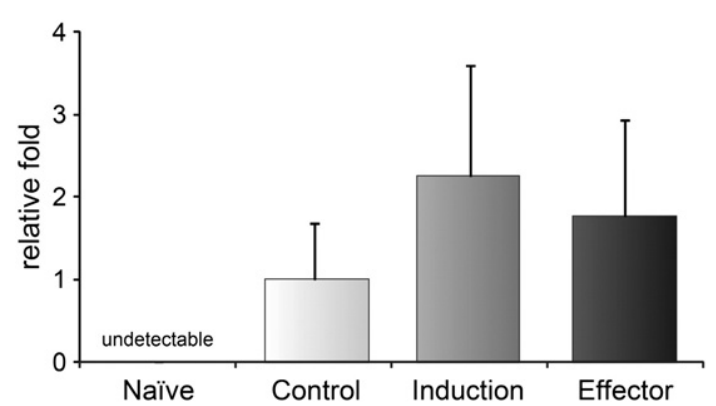

Fig. 5. Semi-quantitative real-time PCR to measure the mRNA expression of TMEV in whole spinal cord. TMEV mRNA expression in the mice treated with anti-PD-1 mAb in either induction or effector phase were comparable to the control IgG-treated mice.

on activated T and B cells (Greenwald et al., 2005). PD-1 interacts with at least two ligands: PD-L1 and another closely related molecule, PD-L2 (Freeman et al., 2000; Latchman et al., 2001; Ishida et al., 2002). The expression patterns of PD-L1 and PD-L2 are largely different. PD-L1 is broadly expressed on leukocytes, nonhematopoietic cells, and in nonlymphoid tissues including islets (Freeman, Long, 2000; Yamazaki et al., 2002; Brown et al., 2003), while PD-L2 is expressed exclusively on DCs and monocytes (Freeman, Long, 2000; Ishida, Iwai, 2002; Ansari et al., 2003; Loke and Allison, 2003). Signaling through PD-1 limits T-cell functions including IFN- $\gamma$ production, proliferation, and increased T-cell apoptosis (Baecher-Allan et al., 2001; Sandner et al., 2005). Notably, negative signals through PD-1 on T cells control autoimmunity. Disruption of the gene encoding PD-1 in BALB/c mice resulted in autoimmune cardiomyopathy (Nishimura et al., 2001), while C57BL/6 PD-1 knockout (KO) mice developed progressive arthritis and lupus like glomerulonephritis disease (Nishimura et al., 1999), and NOD PD-1 KO mice developed accelerated autoimmune diabetes (Wang et al., 2005). The role of PD-1 and its ligands has been further investigated in autoimmune models such as diabetes and multiple sclerosis using blocking reagents. Administration of anti-PD-L1 mAb to young NOD mice resulted in rapid onset of diabetes (Ansari, Salama, 2003). Moreover, PD-1 plays a critical role in murine experimental autoimmune encephalomyelitis (EAE), a mouse model for
MS. In EAE, PD-1 blockade resulted in accelerated and more severe clinical disease (Salama et al., 2003).

We here demonstrated that the expression of PD-1 and PD-L1 on APCs is up-regulated in BMDCs following TMEV infection (Fig. 1). Furthermore, in the spinal cord, the expression of PD-1 and PD-L1 was significantly increased at day 10 post infection. This indicated that TMEV infection induced up-regulation of PD-1 pathway, and this upregulation might contribute to the establishment of persistent infection of TMEV in the CNS. However, in the induction phase, blockade of PD-1 pathway with anti-PD-1 mAb did not affect viral persistence or development of demyelinating disease (Figs. 2, 3, and 4). This suggests that PD-1 pathway alone is not sufficient to establish TMEV persistence in the CNS, and thus, other mechanisms are likely involved.

We have also demonstrated that the expression of PD-1 and PD-L1 was up-regulated at days 20,30 , and 40 post infection when the mice infected with TMEV showed clinical signs and demyelination in the CNS. In this study, we also administrated anti-PD-1 mAb into mice infected with TMEV to block the PD-1 pathway during the effector phase. In consequence, the production of IFN- $\gamma$ and TNF- $\alpha$ by Th1 cells was dramatically increased in the spinal cord and spleen (Figs. 6 and 7). The clinical course of disease was significantly exacerbated by the blockade of PD-1 pathway. Furthermore, IL-17-producing Th17 cells were significantly increased in the spleen of mice treated with anti-PD-1 mAb compared to control mice. Previous studies have shown that IL-17-producing cells are also involved in inflammatory tissue damage, leading to the pathogenesis of various autoimmune diseases (Ouyang et al., 2008) and TMEV-IDD (Hou et al., 2009). Our data suggests that up-regulation of IL-17-producing Th17 cells after blocking the PD-1 pathway might also contribute to the deterioration of TMEVIDD.

We have examined viral persistence levels in the spinal cord by realtime RT-PCR. Viral persistence in the spinal cord of mice treated with anti-PD-1 mAb was comparable to control mice (Fig. 5). This shows that administration of anti-PD- $1 \mathrm{mAb}$ did not affect the viral load in

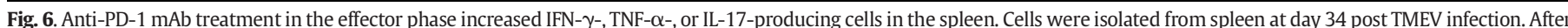

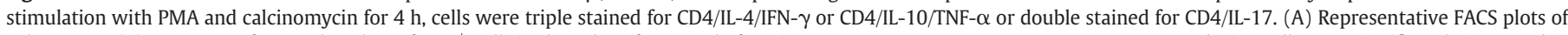

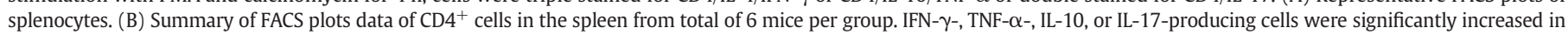
the mice treated with anti-PD-1 mAb in effector phase as compared to the control IgG-treated mice $\left({ }^{*} \mathrm{p}<0.05\right)$. 
A
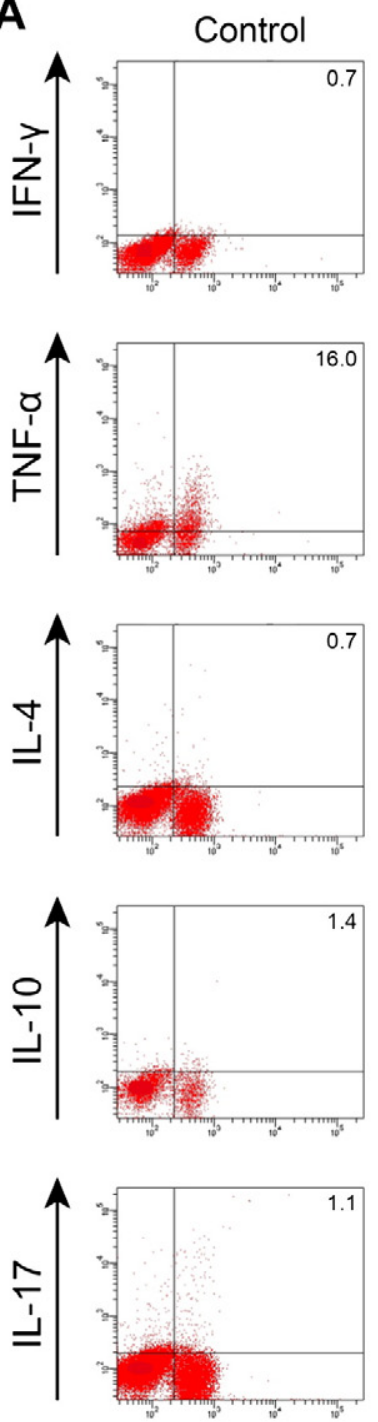

Induction
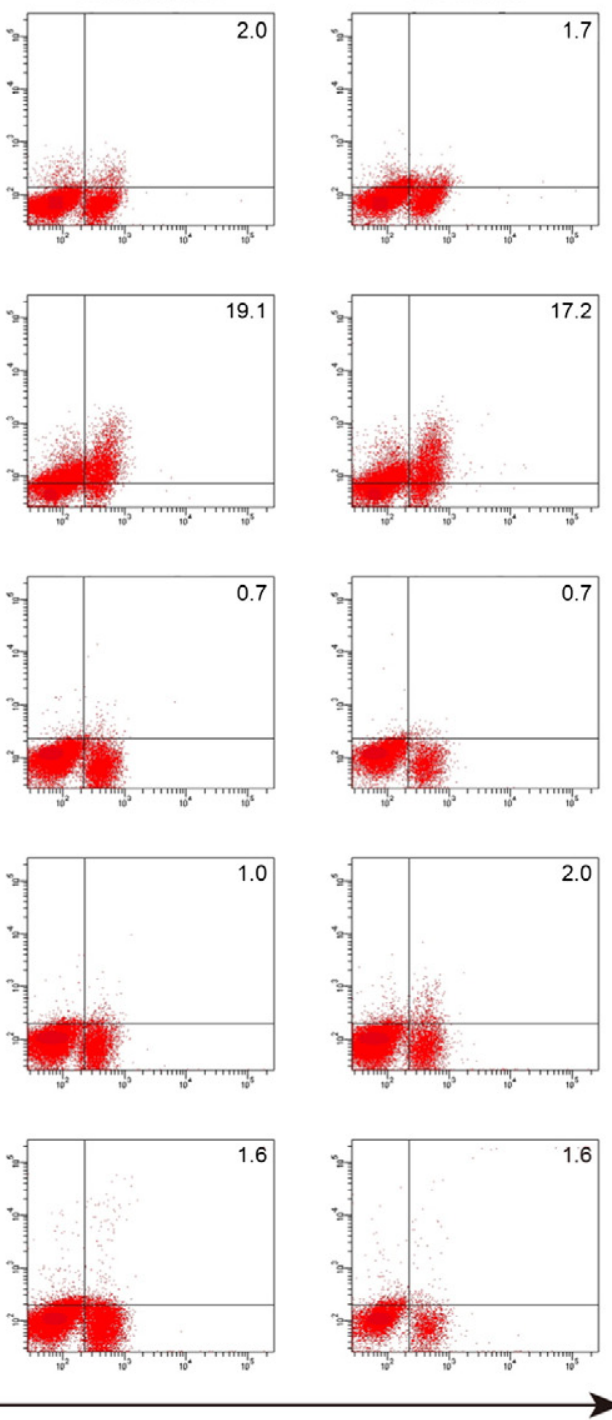

CD4
Effector
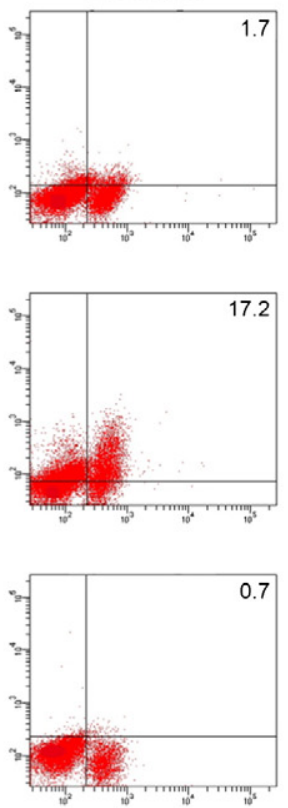

0

B
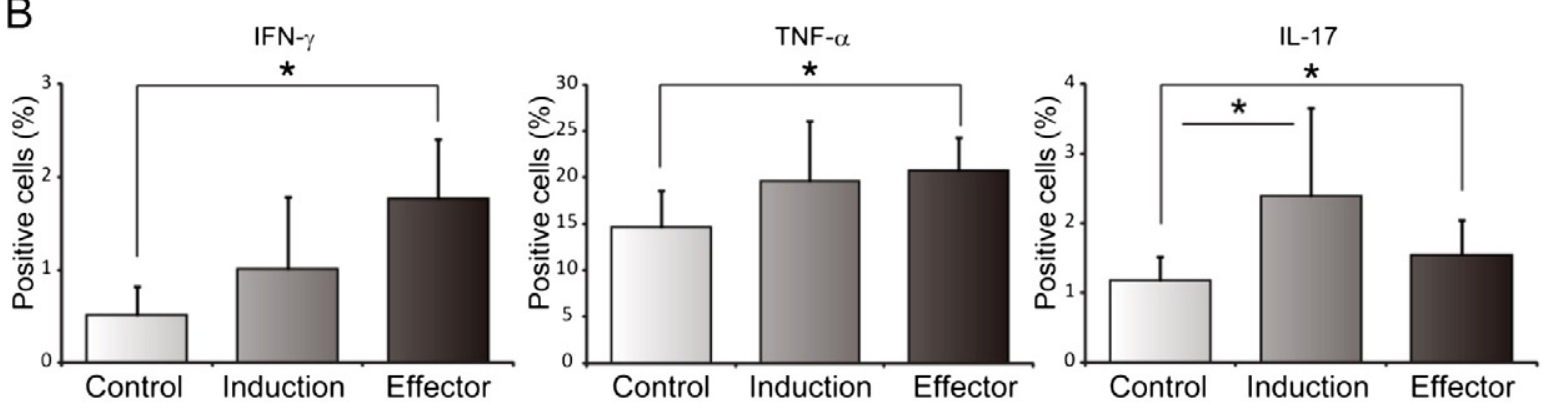

IL-4

IL-10
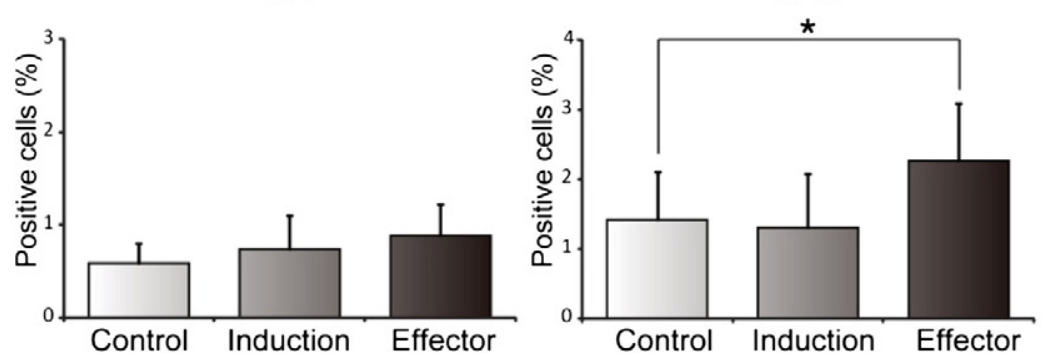
A

A Control

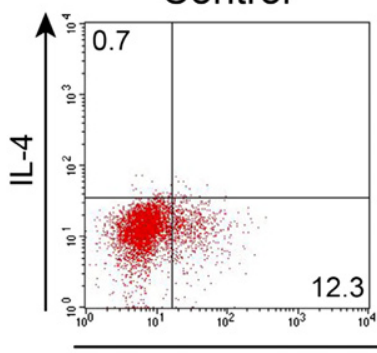

Induction
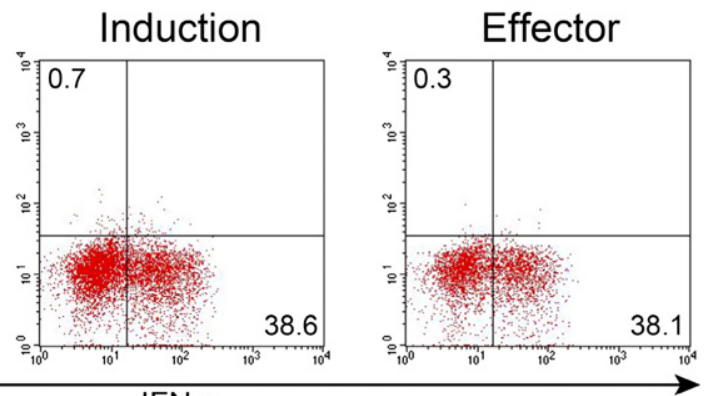

IFN-Y


TNF- $\alpha$

\section{B}
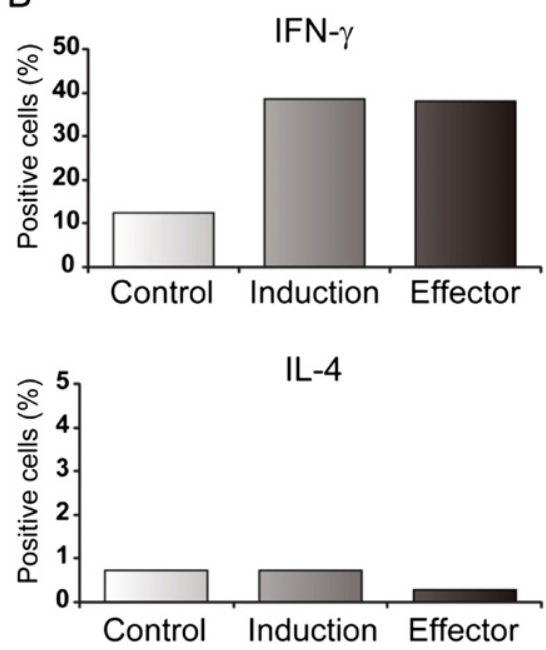

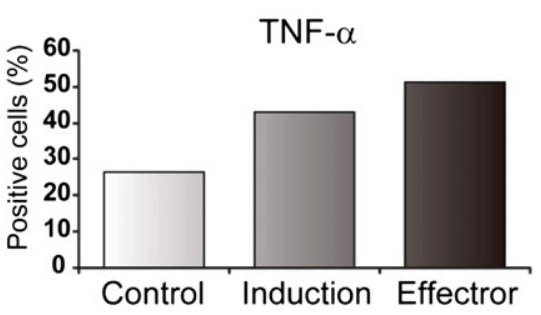

IL-10

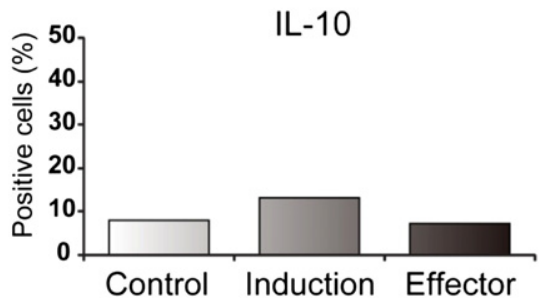

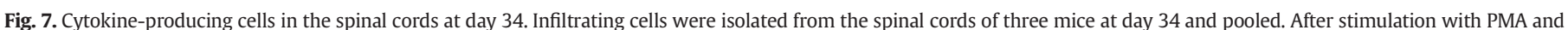


of $\mathrm{CD}^{+}$cells in the spinal cords. IFN- $\gamma$ - or TNF- $\alpha$-producing cells were increased in the mice treated with anti-PD- $1 \mathrm{mAb}$ compared to the control IgG-treated mice.

the CNS, despite the deteriorated disease development. There are increasing evidences that viral persistence levels are not associated with the development of TMEV-IDD (Myoung et al., 2008; Jin et al., 2009). Viral persistence alone may not be sufficient to induce the disease and that the level of $\mathrm{T}$ cell immunity to viral capsid epitopes may be critical for the development of demyelinating disease in SJL mice (Myoung, Bahk, 2008).

In summary, administration of anti-PD-1 mAb during effector phase exacerbated TMEV-IDD. Blockade of PD-1 pathway affected the development of IFN- $\gamma$-producing Th1 cells. TMEV-IDD is considered to be a Th1 disease, and Th1 is also involved in human MS. Therefore, our data suggests the PD-1 pathway may play a critical role in regulating disease onset and progress of virus-induced demyelinating diseases. There is a report that interactions between PD-1/PD-L1, but not PD-1/PDL-2, are crucial in attenuating $\mathrm{T}$ cell responses in EAE (Carter et al., 2007). Therefore, we speculate that blockade of PD-1/PD-L1 pathway is more critical for development of TMEV-IDD than PD-1/PD-L2 pathway. Taken together, PD-1 pathway could be a novel target in the treatment of MS.

\section{Acknowledgments}

This work was supported in part by Health and Labour Sciences Research Grants for research on intractable diseases from Ministry of Health, Labour and Welfare of Japan, and grants from Terumo Lifescience Foundation and Mitsubishi Pharma Research Foundation. We thank Mr. Susumu Itoh for his great technical assistance.

\section{References}

Allen, I., Brankin, B., 1993. Pathogenesis of multiple sclerosis-the immune diathesis and the role of viruses. J. Neuropathol. Exp. Neurol. 52, 95-105.

Ansari, M.J., Salama, A.D., Chitnis, T., Smith, R.N., Yagita, H., Akiba, H., et al., 2003. The programmed death-1 (PD-1) pathway regulates autoimmune diabetes in nonobese diabetic (NOD) mice. J. Exp. Med. 198, 63-69.

Baecher-Allan, C., Brown, J.A., Freeman, G.J., Hafler, D.A., 2001. CD4 + CD25high regulatory cells in human peripheral blood. J. Immunol. 167, 1245-1253.

Brahic, M., Stroop, W.G., Baringer, J.R., 1981. Theiler's virus persists in glial cells during demyelinating disease. Cell 26, 123-128. 
Brown, J.A., Dorfman, D.M., Ma, F.R., Sullivan, E.L., Munoz, O., Wood, C.R., et al., 2003. Blockade of programmed death-1 ligands on dendritic cells enhances T cell activation and cytokine production. J. Immunol. 170, 1257-1266.

Carter, L.L., Leach, M.W., Azoitei, M.L., Cui, J., Pelker, J.W., Jussif, J., et al., 2007. PD-1/PD-L1, but not PD-1/PD-L2, interactions regulate the severity of experimental autoimmune encephalomyelitis. J. Neuroimmunol. 182, 124-134.

Clatch, R.J., Lipton, H.L., Miller, S.D., 1986. Characterization of Theiler's murine encephalomyelitis virus (TMEV)-specific delayed-type hypersensitivity responses in TMEVinduced demyelinating disease: correlation with clinical signs. J. Immunol. 136 920-927.

Clatch, R.J., Miller, S.D., Metzner, R., Dal Canto, M.C., Lipton, H.L., 1990. Monocytes/macrophages isolated from the mouse central nervous system contain infectious Theiler's murine encephalomyelitis virus (TMEV). Virology 176, 244-254.

Dal Canto, M.C., Lipton, H.L., 1975. Primary demyelination in Theiler's virus infection. An ultrastructural study. Lab. Investig. 33, 626-637.

Freeman, G.J., Long, A.J., Iwai, Y., Bourque, K., Chernova, T., Nishimura, H., et al., 2000. Engagement of the PD-1 immunoinhibitory receptor by a novel B7 family member leads to negative regulation of lymphocyte activation. J. Exp. Med. 192, 1027-1034

Friedmann, A., Frankel, G., Lorch, Y., Steinman, L., 1987. Monoclonal anti-I-A antibody reverses chronic paralysis and demyelination in Theiler's virus-infected mice: critical importance of timing of treatment. J. Virol. 61, 898-903.

Gerety, S.J., Rundell, M.K., Dal Canto, M.C., Miller, S.D., 1994. Class II-restricted T cell responses in Theiler's murine encephalomyelitis virus-induced demyelinating disease. VI. Potentiation of demyelination with and characterization of an immunopathologic CD4 + T cell line specific for an immunodominant VP2 epitope. J Immunol 152, 919-929.

Greenwald, R.J., Freeman, G.J., Sharpe, A.H., 2005. The B7 family revisited. Annu. Rev. Immunol. 23, 515-548

Hou, W., Kang, H.S., Kim, B.S., 2009. Th17 cells enhance viral persistence and inhibit T cell cytotoxicity in a model of chronic virus infection. J Exp Med. 206, 313-328.

Ishida, M., Iwai, Y., Tanaka, Y., Okazaki, T., Freeman, G.J., Minato, N., et al., 2002. Differential expression of PD-L1 and PD-L2, ligands for an inhibitory receptor PD-1, in the cells of lymphohematopoietic tissues. Immunol. Lett. 84, 57-62.

Jin, Y.H., Kang, B., Kim, B.S., 2009. Theiler's virus infection induces a predominant pathogenic CD $4+$ T cell response to RNA polymerase in susceptible SJL/J mice. J. Virol. 83, 10981-10992.

Kappel, C.A., Dal Canto, M.C., Melvold, R.W., Kim, B.S., 1991. Hierarchy of effects of the MHC and T cell receptor beta-chain genes in susceptibility to Theiler's murine encephalomyelitis virus-induced demyelinating disease. J. Immunol. 147, 4322-4326.

Karpus, W.J., Pope, J.G., Peterson, J.D., Dal Canto, M.C., Miller, S.D., 1995. Inhibition of Theiler's virus-mediated demyelination by peripheral immune tolerance induction. J. Immunol. 155, 947-957.

Keir, M.E., Butte, M.J., Freeman, G.J., Sharpe, A.H., 2008. PD-1 and its ligands in tolerance and immunity. Annu. Rev. Immunol. 26, 677-704.

Kim, B.S., Yauch, R.L., Bahk, Y.Y., Kang, J.A., Dal Canto, M.C., Hall, C.K., 1998. A spontaneous low-pathogenic variant of Theiler's virus contains an amino acid substitution within the predominant VP1(233-250) T-cell epitope. J. Virol. 72, 1020-1027.

Kurtzke, J.F., Hyllested, K., 1986. Multiple sclerosis in the Faroe Islands. II. Clinical update, transmission, and the nature of MS. Neurology 36, 307-328.

Latchman, Y., Wood, C.R., Chernova, T., Chaudhary, D., Borde, M., Chernova, I., et al., 2001. PD-L2 is a second ligand for PD-1 and inhibits T cell activation. Nat. Immunol. 2 261-268.

Lehrich, J.R., Arnason, B.G., Hochberg, F.H., 1976. Demyelinative myelopathy in mice induced by the DA virus. J. Neurol. Sci. 29, 149-160.

Lipton, H.L., Dal Canto, M.C., 1976. Chronic neurologic disease in Theiler's virus infection of SJL/J mice. J. Neurol. Sci. 30, 201-207.

Lipton, H.L., Kratochvil, J., Sethi, P., Dal Canto, M.C., 1984. Theiler's virus antigen detected in mouse spinal cord 2 1/2 years after infection. Neurology 34, 1117-1119.

Lipton, H.L., Calenoff, M., Bandyopadhyay, P., Miller, S.D., Dal Canto, M.C., Gerety, S., et al 1991. The 5' noncoding sequences from a less virulent Theiler's virus dramatically attenuate GDVII neurovirulence. J. Virol. 65, 4370-4377.

Lipton, H.L., Twaddle, G., Jelachich, M.L., 1995. The predominant virus antigen burden is present in macrophages in Theiler's murine encephalomyelitis virus-induced demyelinating disease. J. Virol. 69, 2525-2533.

Lohmann, T., Leslie, R.D., Londei, M., 1996. T cell clones to epitopes of glutamic acid decarboxylase 65 raised from normal subjects and patients with insulin-dependent diabetes. J. Autoimmun. 9, 385-389.

Loke, P., Allison, J.P., 2003. PD-L1 and PD-L2 are differentially regulated by Th1 and Th2 cells. Proc. Natl. Acad. Sci. U. S. A. 100, 5336-5341.

Miller, S.D., Vanderlugt, C.L., Begolka, W.S., Pao, W., Neville, K.L., Yauch, R.L., et al., 1997a. Epitope spreading leads to myelin-specific autoimmune responses in SJL mice chronically infected with Theiler's virus. J. Neurovirol. 3 (Suppl. 1), S62-S65.
Miller, S.D., Vanderlugt, C.L., Begolka, W.S., Pao, W., Yauch, R.L., Neville, K.L., et al., 1997b. Persistent infection with Theiler's virus leads to CNS autoimmunity via epitope spreading. Nat. Med. 3, 1133-1136.

Myoung, J., Bahk, Y.Y., Kang, H.S., Dal Canto, M.C., Kim, B.S., 2008. Anticapsid immunity level, not viral persistence level, correlates with the progression of Theiler's virusinduced demyelinating disease in viral P1-transgenic mice. J. Virol. 82, 5606-5617.

Nathanson, N., Miller, A., 1978. Epidemiology of multiple sclerosis: critique of the evidence for a viral etiology. Am. J. Epidemiol. 107, 451-461.

Nishimura, H., Nose, M., Hiai, H., Minato, N., Honjo, T., 1999. Development of lupus-like autoimmune diseases by disruption of the PD-1 gene encoding an ITIM motifcarrying immunoreceptor. Immunity $11,141-151$.

Nishimura, H., Okazaki, T., Tanaka, Y., Nakatani, K., Hara, M., Matsumori, A., et al., 2001. Autoimmune dilated cardiomyopathy in PD-1 receptor-deficient mice. Science 291, 319-322.

Nurieva, R.I., Liu, X., Dong, C., 2009. Yin-Yang of costimulation: crucial controls of immune tolerance and function. Immunol. Rev. 229, 88-100.

Okazaki, T., Honjo, T., 2007. PD-1 and PD-1 ligands: from discovery to clinical application. Int. Immunol. 19, 813-824.

Ouyang, W., Kolls, J.K., Zheng, Y., 2008. The biological functions of T helper 17 cell effector cytokines in inflammation. Immunity 28, 454-467.

Peterson, J.D., Karpus, W.J., Clatch, R.J., Miller, S.D., 1993. Split tolerance of Th1 and Th2 cells in tolerance to Theiler's murine encephalomyelitis virus. Eur. J. Immunol. 23, $46-55$.

Pevear, D.C., Calenoff, M., Rozhon, E., Lipton, H.L., 1987. Analysis of the complete nucleotide sequence of the picornavirus Theiler's murine encephalomyelitis virus indicates that it is closely related to cardioviruses. J. Virol. 61, 1507-1516.

Rodriguez, M., Lafuse, W.P., Leibowitz, J., David, C.S., 1986. Partial suppression of Theiler's virus-induced demyelination in vivo by administration of monoclonal antibodies to immune-response gene products (la antigens). Neurology 36, 964-970.

Salama, A.D., Chitnis, T., Imitola, J., Ansari, M.J., Akiba, H., Tushima, F., et al., 2003. Critical role of the programmed death-1 (PD-1) pathway in regulation of experimental autoimmune encephalomyelitis. J. Exp. Med. 198, 71-78.

Sandner, S.E., Clarkson, M.R., Salama, A.D., Sanchez-Fueyo, A., Domenig, C., Habicht, A., et al., 2005. Role of the programmed death-1 pathway in regulation of alloimmune responses in vivo. J. Immunol. 174, 3408-3415.

Sharpe, A.H., 2009. Mechanisms of costimulation. Immunol. Rev. 229, 5-11.

Sharpe, A.H., Wherry, E.J., Ahmed, R., Freeman, G.J., 2007. The function of programmed cell death 1 and its ligands in regulating autoimmunity and infection. Nat. Immunol. 8, 239-245.

Takeichi, N., Yanagisawa, S., Kaneyama, T., Yagita, H., Jin, Y.H., Kim, B.S., et al., 2010. Ameliorating effects of anti-Dll4 mAb on Theiler's murine encephalomyelitis virusinduced demyelinating disease. Int. Immunol. 22, 729-738.

Tsugane, S., Takizawa, S., Kaneyama, T., Ichikawa, M., Yagita, H., Kim, B.S., et al., 2012. Therapeutic effects of anti-Delta $1 \mathrm{mAb}$ on Theiler's murine encephalomyelitis virus-induced demyelinating disease. J. Neuroimmunol. 252, 66-74.

van Noort, J.M., van Sechel, A., Boon, J., Boersma, W.J., Polman, C.H., Lucas, C.J., 1993. Minor myelin proteins can be major targets for peripheral blood $\mathrm{T}$ cells from both multiple sclerosis patients and healthy subjects. J. Neuroimmunol. 46, 67-72.

Wang, J., Yoshida, T., Nakaki, F., Hiai, H., Okazaki, T., Honjo, T., 2005. Establishment of NOD-Pdcd $1-/-$ mice as an efficient animal model of type I diabetes. Proc. Natl. Acad. Sci. U. S. A. 102, 11823-11828.

Wekerle, H., 1991. Immunopathogenesis of multiple sclerosis. Acta Neurol. (Napoli) 13, 197-204.

Welsh, C.J., Tonks, P., Nash, A.A., Blakemore, W.F., 1987. The effect of L3T4 T cell depletion on the pathogenesis of Theiler's murine encephalomyelitis virus infection in CBA mice. J. Gen. Virol. 68 (Pt 6), 1659-1667.

Williams, I.R., Unanue, E.R., 1990. Costimulatory requirements of murine Th1 clones. The role of accessory cell-derived signals in responses to anti-CD3 antibody. J. Immunol. $145,85-93$

Yamazaki, T., Akiba, H., Iwai, H., Matsuda, H., Aoki, M., Tanno, Y., et al., 2002. Expression of programmed death 1 ligands by murine T cells and APC. J. Immunol. 169, 5538-5545.

Yamazaki, T., Akiba, H., Koyanagi, A., Azuma, M., Yagita, H., Okumura, K., 2005. Blockade of B7-H1 on macrophages suppresses CD4 + T cell proliferation by augmenting IFNgamma-induced nitric oxide production. J. Immunol. 175, 1586-1592.

Yanagisawa, S., Takeichi, N., Kaneyama, T., Yagita, H., Taniguchi, S., Kim, B.S., et al., 2010. Effects of anti-CD70 mAb on Theiler's murine encephalomyelitis virus-induced demyelinating disease. Brain Res. 1317, 236-245.

Yauch, R.L., Palma, J.P., Yahikozawa, H., Koh, C.S., Kim, B.S., 1998. Role of individual T-cell epitopes of Theiler's virus in the pathogenesis of demyelination correlates with the ability to induce a Th1 response. J. Virol. 72, 6169-6174. 